\title{
Clinical, radiological and histological diagnoses of periapical periodontitis spreading to the adjacent tooth: A case of endodontic failure
}

\author{
Luigi Cianconi, Manuele Mancini
}

Department of Restorative Dentistry and Endodontics, University of Rome “Tor Vergata”, Rome, Italy

Email: manuele.mancini@tiscali.it

Received 23 May 2013; revised 24 June 2013; accepted 15 July 2013

Copyright (c) 2013 Luigi Cianconi, Manuele Mancini. This is an open access article distributed under the Creative Commons Attribution License, which permits unrestricted use, distribution, and reproduction in any medium, provided the original work is properly cited.

\begin{abstract}
Aims: This article describes the apical infection in endodontically treated tooth 4.5 that spread to adjacent tooth 4.4. Case Report: A 52-year-old woman was referred for the presence of radiolucency extending from tooth 4.5 and mental foramen. Spontaneous symptoms were present. Tooth 4.5 showed poor-quality endodontics. The vitality of tooth 4.4 was negative, even though no mechanical trauma had been reported, nor was caries present. Both teeth were sensitive to percussion. Endodontic re-treatment of 4.5 and endodontic treatment of 4.4 were performed in a single visit. A large amount of endodontic sealer squeezed mesially from the root of tooth 4.5 , where a partial horizontal root fracture was hypothesized. 6-, 12-, and 18-month radiographic follow-ups, by both periapical and cone-beam computed tomography (CBCT) analyses, showed incomplete osseous healing. CBCT excluded root fracture on 4.5. Local symptoms were still present. Clinical and radiological conditions led to extractions, and a cystic lesion was enucleated for histopathologic analyses. Histopathologic diagnosis was a periapical cyst. The supposed partial horizontal root fracture of 4.5 was actually a large lateral canal. Although the root canal treatments followed high standards in terms of quality, a persistent chronic infection developed histologically. The cystic lesion was one consistent reason for the unsuccessful healing of 4.5.
\end{abstract}

Keywords: Apical Periodontitis; Apicoectomy; Follow-Up; CBCT; Cyst; Lateral Canal

\section{INTRODUCTION}

The diagnosis of periapical lesions is usually based on clinical and radiographic findings, which are empirical methods. Nevertheless, endodontists commonly strive to make a definitive diagnosis of periapical pathologies of inflammatory origin. A final diagnosis can be achieved by histopathological examination of the tissues, which is not practical in cases of non-surgical treatment. Pulpal necrosis and apical lesion deployment occur only when oral microbiota exists [1,2]. Non-surgical root canal treatment has a very high success rate in teeth with apical periodontitis [3,4]; therefore, a necrotic tooth with apical periodontitis generally receives non-surgical root canal treatment alone. Periapical radiographs provide important information about the development, reduction, and persistence of apical periodontitis, as well as indispensable data from which treatment decisions can be made. Radiographically, inflammatory periapical pathologies present as a radiolucent area, with or without a well-defined periphery simulating a cortex. The presence of a radiopaque cortex, displacement of adjacent structures, and diameter exceeding $9.5 \mathrm{~mm}$ usually allow the clinician to diagnose a periapical cyst $[5,6]$. However, the radiographic image, as a shadow, has the elusive qualities of all shadows. It is a two-dimensional representation of a three-dimensional object. New imaging modalities have been used as diagnostic tools in dental radiology. Conebeam computed tomography (CBCT) produces detailed high-resolution, three-dimensional images of oral structures, which may allow bone lesions to be detected at an early stage $[7,8]$. According to the literature, imaging methods are useful tools for the diagnosis of periapical lesions [9], but are not sufficiently accurate to be used as a standard criterion for diagnosis, as is, in this case, the histological examination of the lesion by microscopy [10].

\section{CASE HISTORY}

A 52-year-old woman was referred to for the presence of 
radiolucency extending from the apex of tooth 4.5 and the mental foramen. The patient's medical history was non-contributory, and spontaneous symptoms (burning sensation and right lower lip numbness) were reported. Nor intraoral neither extraoral swelling was present. A mild response to percussion testing of teeth 4.5 and 4.4 was present. Radiographic examination revealed an inadequately treated root canal associated with a large radiolucent periradicular lesion extending from the apical to the mesial aspect of the root of tooth 4.5 and the apex of tooth 4.4 (Figure 1(A)). Radiopaque root canal filling material was observed at the apical segment of the canal, and radiolucent post-endodontic core material was visible at the coronal third of the canal. Cold, warm, and electric pulp vitality testing on tooth 4.4 was negative. Endodontic re-treatment of tooth 4.5 and endodontic treatment of tooth 4.4 were performed in a single visit, needing no intracanal medicaments, as described by $\mathrm{Su}$ et al. [11]. Antibiotics were not prescripted because nor swelling neither purulent exudate was noticeable.

\subsection{Tooth 4.5}

After removal of the provisional prosthodontic coronal restoration, followed by rubber dam isolation, access to the coronal cavity was completed. The operative field was decontaminated with tincture of iodine $(10 \% \mathrm{w} / \mathrm{v})$ (Betadine; Meda Pharma SpA, Milano, Italy), and an ultrasound Start-X tip \#3 (Dentsply Maillefer, Ballaigues, Switzerland) was used to retrieve the carbon-fiber post luted at the coronal part of the canal. The root canal was instrumented as follows: Gates-Glidden burs (sizes \#2, 3, and 4) were used to enlarge the coronal and middle segments of the root canal. Hand SS K-type files (Dentsply Maillefer) were used until the working length-measured by deducting $0.5 \mathrm{~mm}$ from the measurement on the “APEX” mark of \#15 K-file (Propex II; Dentsply Maillefer) — was reached. Apical preparation was performed with a ProTaper F4 file. Apical patency was maintained throughout the procedures by a \#20 K-type file. Vigorous irrigation with $5.25 \% 37^{\circ} \mathrm{C} \mathrm{NaOCl}$ was performed after each file. The smear layer was removed by the use of 5 $\mathrm{mL} 17 \%$ EDTA followed by $5.25 \% 37^{\circ} \mathrm{C} \mathrm{NaOCl}$. The root canal was dried with sterile paper-points and filled by the Continuous Wave Technique with gutta-percha and AH Plus Sealer (Dentsply DeTrey, Konstanz, Germany). A large lateral canal, initially judged as a partial horizontal root fracture, was revealed after obturation (Figure 1(B)). The coronal cavity was restored with composite.

\subsection{Tooth 4.4}

Endodontic access to the pulp chamber was gained with a round diamond-coated bur (Dentsply Maillefer). Pulp canal debris was removed from the coronal third of the canal with a \#4 Gates Glidden bur. We measured the working length by deducting $0.5 \mathrm{~mm}$ from the measurement on the "APEX" mark of \#15 K-file (Dentsply Maillefer). The tooth was shaped by means of ProTaper Ni-Ti rotary instruments (Dentsply Maillefer), according to the manufacturer's instructions, until the F4 file reached the working length. Apical patency was maintained throughout the procedures by a \#20 K-type file. Vigorous irrigation with $5.25 \% 37^{\circ} \mathrm{C} \mathrm{NaOCl}$ was performed after each file. The smear layer was removed by the use of $5 \mathrm{~mL} 17 \%$ EDTA followed by $5.25 \% 37^{\circ} \mathrm{C}$ $\mathrm{NaOCl}$. The root canal was dried with sterile paperpoints and filled by the Continuous Wave Technique with gutta-percha and AH Plus Sealer (Dentsply DeTrey). The coronal cavity was restored with composite (Figure 1(B)).

\subsection{Follow-Up}

Six-month radiographic follow-up showed incomplete osseous healing. Symptoms were still present on both teeth. Most of the sealer extruding periapically and laterally was not resorbed (Figure 1(C)). Local clinical symptoms (burning sensation and lip numbness) were still present. The periapical X-ray 12 months after endodontic therapies showed a radiolucent periapical lesion; therefore, a CBCT analysis was prescribed to exclude a partial horizontal root fracture on tooth 4.5 (Figure 1(D)). The sealer extruding laterally on tooth 4.5 was still visible, whereas only minimal residual sealer could be seen on the apical aspect of tooth 4.4. At this stage no further therapies were scheduled, in accordance to studies showing no benefits would have been obtained [3,4]. In the follow-up appointment 18 months after re-treatment/treatment, spontaneous clinical symptoms were present, teeth were still symptomatic, and a new CBCT analysis revealed periradicular radiolucency suggestive of persistent disease (Figure 2(A)). The time elapsed since the end of the therapy, as described by some Authors [3,4], the good quality of the re-treatment, and the presence of symptomatic pain led us to perform apicoectomy surgery on teeth 4.4 and 4.5. The patient participated in the decision-making process. Treatment planning changed during surgery, however. The dental surgeon decided to extract both teeth for two reasons: first, because the cystic lesion included tooth 4.4 and was very close to the mental foramen (Figure 2(B)), thus explaining the patient's continuous and spontaneous pain; and second, because the root remaining after apicoectomy behind the supposed partial fracture of root 4.5 would have not withstood post-endodontic treatment and occlusal load. The cystic lesion was processed for histopathologic analyses. 

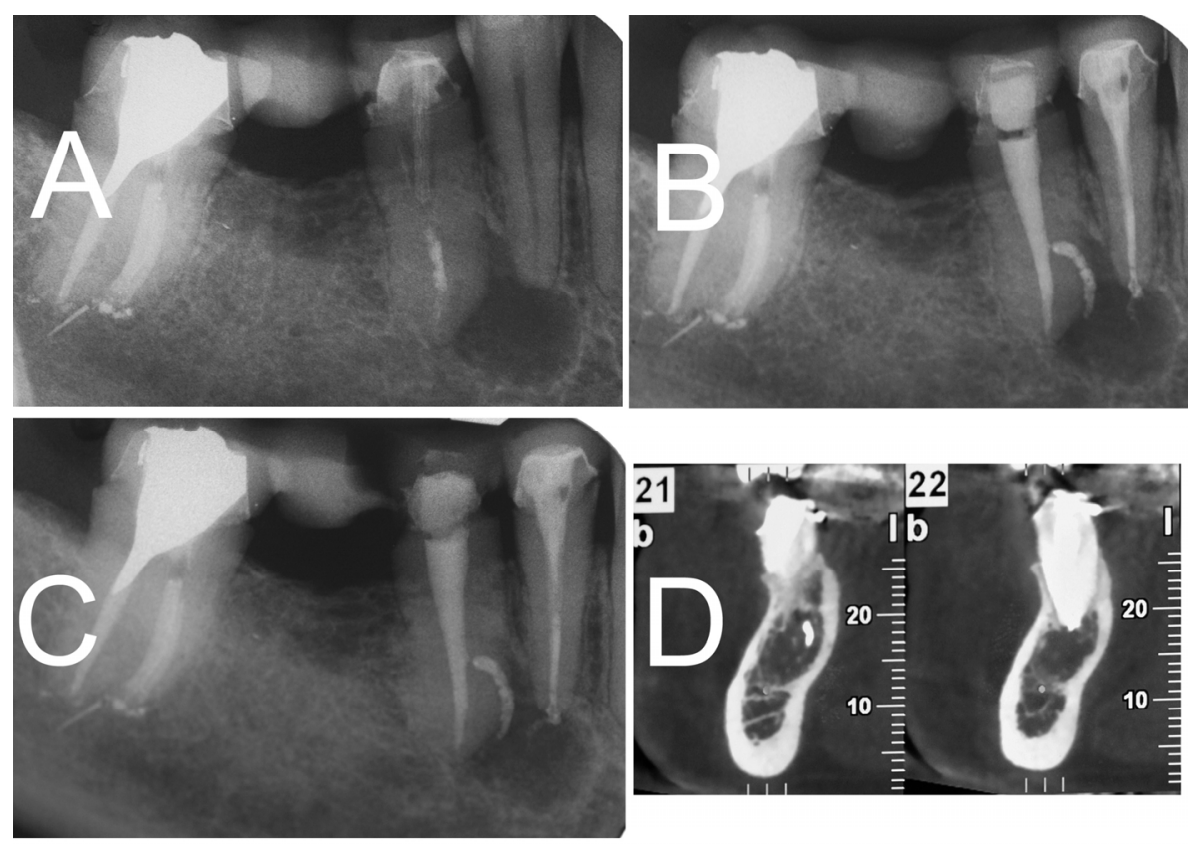

Figure 1. (A) Periapical x-ray showing a large radiolucent periradicular lesion extending from the apical to the mesial aspect of the root of tooth 4.5 and the apex of tooth 4.4; (B) Periapical X-ray showing a large lateral canal on tooth 4.5 after the endodontic obturation; (C) Six-month radiographic follow-up showed incomplete osseous healing. Most of the sealer extruded periapically and laterally on tooth 4.5 was not resorbed; (D) Twelve-month radiographic follow-up: CBCT analysis was prescribed to exclude a partial horizontal root fracture on tooth 4.5 .

\section{SPECIMEN ANALYSIS}

\subsection{Specimen Preparation}

Specimens were immediately immersed in $10 \%$ neutral buffered formalin and fixed for 48 hours. Care was taken to enucleate the lesion still attached to the root tip. Three-week demineralization was carried out in an aqueous solution consisting of a mixture of $22.5 \%$ (vol/vol) formic acid and 10\% (wt/vol) sodium citrate. The biopsy specimen was washed in running water for 48 hours, dehydrated in ascending grades of ethanol, cleared in xylene, and infiltrated and embedded in paraffin (melting point $56^{\circ} \mathrm{C}$ ) according to standard procedures.

\subsection{Histopathological Analysis}

The pathology report revealed a fibrous, hyperplastic, non-keratinized squamous tissue with bony spicules, and a site of chronic inflammation corresponding to activated epithelial cell rests of Malassez (Figure 2(C)). The histopathologic diagnosis was a periapical cyst. The supposed partial horizontal root fracture of tooth 4.5 was actually a large lateral canal (Figure 2(D)).

\section{DISCUSSION}

Many authors have demonstrated that from 94\% to 99\% of periapical lesions are associated with pulpal disease and diagnosed as periapical granulomas, cysts, or abscesses [12]. Biopsies of periapical lesions are usually undertaken for diagnostic affirmation, persistent or atypical presentations, or for cases with a relevant medical history [13]. The differential diagnosis of periapical radiolucencies is diverse [14]. Post-treatment apical periodontitis is caused by either persistent or secondary intraradicular infection [15]. Micro-organisms that persist after intracanal procedures of disinfection and manage to survive in the obturated root canal can cause persistent infections. Secondary infections are usually caused by bacteria introduced into the canal via a breach in asepsis during treatment or via coronal leakage in obturated root canals exposed to the oral cavity. Post-treatment apical periodontitis can also be categorized as recurrent (redeveloping after having healed) [15]. Recurrent disease quite often represents a late failure of the endodontic treatment, and the cause is conceivably related to a new event arising years after treatment conclusion. Coronal leakage after tooth fracture or loss of the permanent coronal restoration might be an example of such a new event [16]. Bacteria causing persistent infections are usually located in areas unaffected by instruments and antimicrobial substances, including lateral canals, apical ramifications, and isthmuses [17]. In addition, bacteria may even remain in the main canal, especially on dentinal 


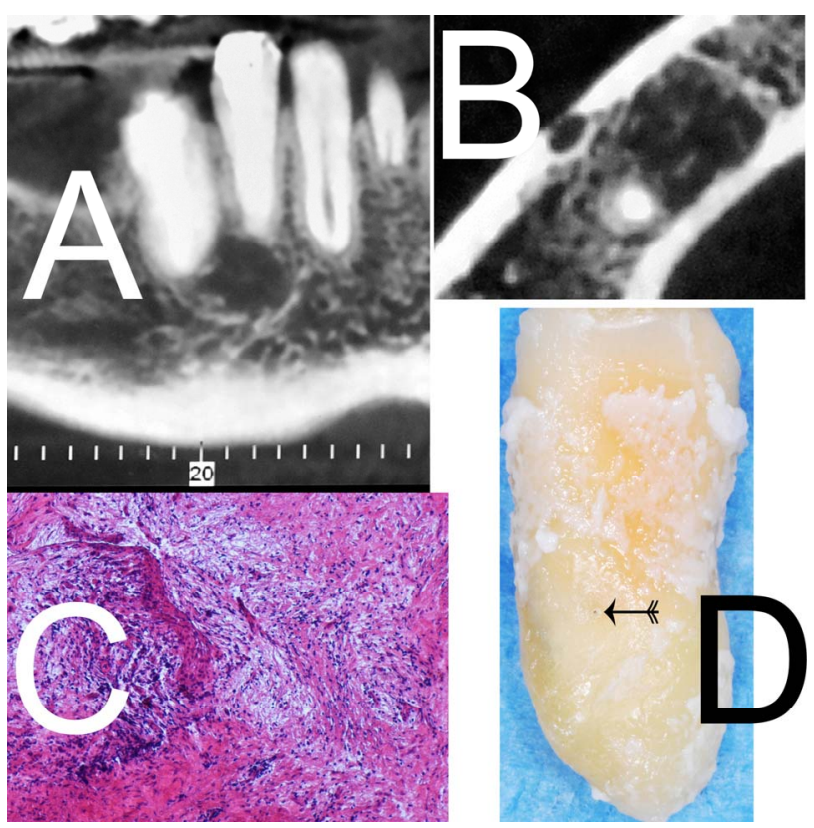

Figure 2. (A) Eighteen months CBCT analysis after retreatment/treatment revealed periradicular radiolucency suggestive of persistent disease; (B) Eighteen months CBCT analysis showing lesion close to the mental foramen; (C) Histopathology image showing fibrous, hyperplastic, nonkeratinized squamous tissue; (D) The large lateral canal filled with endodontic sealer on the mesial aspect of tooth 4.5 .

canal walls that remained untouched by instruments [18]. Bacterial invasion of dentinal tubules has also been regarded as a potential source of persistent infection [19], but there is no convincing report on dentinal tubule infection as a cause of post-treatment disease [20].

This article describes a radiolucency involving two teeth and the failure of non-surgical endodontic root canal therapies. Tooth 4.5 had necrotic pulp with apical periodontitis, whereas tooth 4.4 had necrotic pulp with normal apical tissue. No caries, trauma, or fracture was observed on tooth 4.4. It was hypothesized that the persistent apical lesion and infection on tooth 4.5 affected tooth 4.4. Tooth 4.5 did not respond to re-treatment, perhaps because of coronal leakage or insufficient intracanal cleaning and shaping at the apical area, which resulted in an insufficient filling. Bacteria growing in a poor coronal filling can increase significantly, gain access to the apical lesion, and continue to inflame and infect the apical tissues [21]. Post-treatment apical periodontitis is primarily an infectious disease caused by either an intraradicular or an extraradicular infection [21]. In treated teeth with posttreatment disease in which the canals were apparently treated under acceptable standards, bacteria are usually observed in biofilms colonizing untouched areas of the main root canal, apical ramifications, lateral canals, and isthmuses [22]. Although the root canal was instrumented up to its terminus, a strong concentration (5.25\%) of $\mathrm{NaOCl}$ was used for irrigation, a large amount of sealer was squeezed into a large lateral canal, and the obturation followed a high standard in terms of quality (apical length and homogeneity), a persistent chronic infection still developed histologically. Stashenko et al. [23] showed that apical infections might spread and produce symptoms when bacteria become stronger than the host's defense systems. Also, the anatomical complexities of the apical area may limit treatment success [24]. One consistent reason for the unsuccessful healing on tooth 4.5 was the cystic lesion, which expanded laterally to tooth 4.4 due to the thickness of the cortical bone and the thin anatomical structure in the lateral teeth of the mandible. According to Skaug [25], fluid pressure in odontogenic jaw cysts is higher than the atmospheric pressure. It has been speculated that increased intracystic fluid pressure might activate the growth of odontogenic jaw cysts [26]. Large cystic and periapical lesions are difficult to heal with only non-surgical root canal treatment [27]. Radiographs (Figures 1(D) and 2(A)) show a welldefined circumscribed radiolucency with a well-defined cortical border, which was interrupted by the apex of tooth number 4.5. During surgery, tissue was found to be attached to the apex of tooth number 4.5 and needed to be separated for biopsy. In summary, this rare case shows that apical infection from one tooth may spread to adjacent teeth.

\section{CONCLUSION}

In this case report, although the root canal treatments followed high standards in terms of quality, the evidencebased radiological and clinical follow-ups were scheduled, a persistent chronic infection developed histologically. The cystic lesion was one consistent reason for the unsuccessful healing of apical-periapical translucency.

\section{REFERENCES}

[1] Kakehashi, S., Stanley, H.R. and Fitzgerald, R.J. (1965) The effects of surgical exposures of dental pulps in germfree and conventional laboratory rats. Oral Surgery, Oral Medicine, Oral Pathology, 20, 340-349. doi:10.1016/0030-4220(65)90166-0

[2] Sundqvist, G. (1976) Bacteriological studies of necrotic dental pulps. University of Umea, Umea.

[3] Sjögren, U., Hägglund, B., Sundqvist, G. and Wing, K. (1990) Factors affecting the long-term results of endodontic treatment. Journal of Endodontics, 16, 498-504. doi:10.1016/S0099-2399(07)80180-4

[4] Marquis, V.L., Dao, T., Farzaneh, M., Abitbol, S. and Friedman, S. (2006) Treatment outcome in endodontics: The Toronto study. Phase III: Initial treatment. Journal of Endodontics, 32, 299-306. doi:10.1016/j.joen.2005.10.050 
[5] McCall, J.O. and Wald, S.S. (1952) Clinical dental roentgenology. 3rd Edition, W.B. Saunders, Philadelphia.

[6] Grossman, L.I. (1950) Root canal therapy. 3rd Edition, Lea \& Febiger, Philadelphia.

[7] Estrela, C., Bueno, M.R., Leles, C.R., et al. (2008) Accuracy of cone beam computed tomography and panoramic and periapical radiography for detection of apical periodontitis. Journal of Endodontics, 34, 273-279. doi:10.1016/j.joen.2007.11.023

[8] Nair, M.K. and Nair, U.P. (2007) Digital and advanced imaging in endodontics: A review. Journal of Endodontics, 33, 1-6. doi:10.1016/j.joen.2006.08.013

[9] Simon, J. H. S., Enciso, R., Malfaz, J.M., et al. (2006) Differential diagnosis of large periapical lesions using cone beam computed tomography measurements and biopsy. Journal of Endodontics, 32, 833-837. doi:10.1016/j.joen.2006.03.008

[10] Rosenberg, P.A., Frisbie, J., Lee, J., et al. (2010) Evaluation of pathologists (histopathology) and radiologists (cone beam computed tomography) differentiating radicular cysts from granulomas. Journal of Endodontics, 36, 423-428. doi:10.1016/j.joen.2009.11.005

[11] Su, Y., Wang, C. and Ye, L. (2011) Healing rate and post-obturation pain of single- versus multiple-visitendodontic treatment for infectedrootcanals: A systematic review. Journal of Endodontics, 37, 125-132. doi:10.1016/j.joen.2010.09.005

[12] Peters, E. and Lau, M. (2003) Histopathologic examination to confirm diagnosis of periapical lesions: A review. Journal of the Canadian Dental Association, 69, 598600.

[13] Newton, C.W. (1999) To biopsy or not [letter]. Oral Surgery, Oral Medicine, Oral Pathology, Oral Radiology, and Endodontics, 87, 642-643. doi:10.1016/S1079-2104(99)70177-0

[14] Brooks, J.K., Ribera, M.J., Rogers, C.W., et al. (2005) Radiographic challenges: Report of 5 cases. New York State Dental Journal, 71, 48-50.

[15] Siqueira Jr., J.F. (2011) Treatment of endodontic infections. Quintessence Publishing, London.

[16] Ricucci, D. and Siqueira Jr., J.F. (2011) Recurrent apical periodontitis and late endodontic treatment failure related to coronal leakage: A case report. Journal of Endodontics, 37, 1171-1175. doi:10.1016/j.joen.2011.05.025
[17] Ricucci, D. and Siqueira Jr., J.F. (2010) Biofilms and apical periodontitis: Study of prevalence and association with clinical and histopathologic findings. Journal of Endodontics, 36, 1277-1288. doi:10.1016/j.joen.2010.04.007

[18] Peters, O.A., Schönenberger, K. and Laib, A. (2001) Effects of four Ni-Ti preparation techniques on root canal geometry assessed by micro computed tomography. International Endodontic Journal, 34, 221-230.

[19] Love, R.M. and Jenkinson, H.F. (2002) Invasion of dentinal tubules by oral bacteria. Critical Reviews in Oral Biology \& Medicine, 13, 171-183. doi:10.1177/154411130201300207

[20] Peters, L.B., Wesselink, P.R. and Moorer, W.R. (1995) The fate and the role of bacteria left in root dentinal tubules. International Endodontic Journal, 28, 95-99. doi:10.1111/j.1365-2591.1995.tb00166.x

[21] Siqueira, J.F. (2001) Aetiology of root canal treatment failure: Why well treated teeth can fail. International Endodontic Journal, 34, 1-10. doi:10.1046/j.1365-2591.2001.00396.x

[22] Lin, L.M., Skribner, J.E. and Gaengler, P. (1992) Factors associated with endodontic treatment failures. Journal of Endodontics, 18, 625-627. doi:10.1016/S0099-2399(06)81335-X

[23] Stashenko, P., Wang, C.-Y., Tani-Ishii, N. and Yu, S.M. (1994) Pathogenesis of induced rat periapical lesions. Oral Surgery, Oral Medicine, Oral Pathology, 78, 494 502. doi:10.1016/0030-4220(94)90044-2

[24] Ricucci, D. and Siqueira Jr., J.F. (2008) Anatomic and microbiologic challenges to achieving success with endodontic treatment: A case report. Journal of Endodontics, 34, 1249-1254. doi:10.1016/j.joen.2008.07.002

[25] Skaug, N. (1976) Intracystic fluid pressure in non-keratinizing jaw cysts. International Journal of Oral Surgery, 5, 59-65. doi:10.1016/S0300-9785(76)80049-X

[26] Oka, S., Kubota, Y., Yamashiro, T., Ogata, S., Ninomiya, T., Ito, S., et al. (2005) Effects of positive pressure in odontogenic keratocysts. Journal of Dental Research, 84, 913-918. doi:10.1177/154405910508401008

[27] Nair, P.N. (1998) New perspectives on radicular cysts: Do they heal? International Endodontic Journal, 31, 155160. doi:10.1046/j.1365-2591.1998.00146.x 